\section{Plasticité des cellules cancéreuses et dissémination métastatique}

Caroline Moyret-Lalle, Roxane Pommier, Charlotte Bouard, Ebticem Nouri, Geoffrey Richard, Alain Puisieux

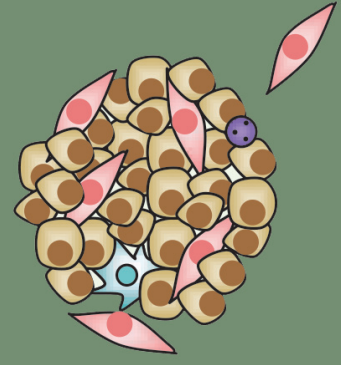

Université de Lyon, Université Claude Bernard Lyon 1, Inserm 1052, CNRS 5286, Centre Léon Bérard, Centre de recherche en cancérologie de Lyon, 28, rue Laënnec, 69008 Lyon, France. alain.puisieux@lyon.unicancer.fr

manière individuelle ou collective, dans le tissu conjonctif intra- et péritumoral, entraînant une invasion locale. Cette étape précoce peut être dissociée des suivantes puisqu'une tumeur invasive localement ne devient pas forcément métastatique à distance. Le processus d'intravasation constitue une étape critique de la dissémination des cellules tumorales au sein de l'organisme puisqu'elle consiste en la traversée de la paroi des vaisseaux sanguins ou lymphatiques par les cellules cancéreuses pour entrer dans la circulation. Ce phénomène est favorisé par l'angiogenèse d'origine tumorale ou «néo-angiogenèse », c'est-à-dire la formation de nouveaux vaisseaux capables d'irriguer la tumeur en développement [39] $(\rightarrow)$.

Les cellules qui survivent aux pressions physiques $(\rightarrow)$ Voir la Synthèse et aux contraintes soumises par la circulation pourront procéder par la suite à l'étape d'extravasion, la sortie des vaisseaux leur permettant de L. Treps et J. Gavard, $m / s n^{\circ} 11$, novembre 2015, page 989 au développement de métastases, le processus de dissémination métastatique des cellules cancéreuses reste mal connu [43] $(\rightarrow)$. Cette relative méconnaissance traduit

$(\rightarrow)$ Voir la Nouvelle de A. Puisieux et S. Ansieau, $m / s n^{\circ} 1$, janvier 2009, page 28 nomène. II s'agit d'un processus d'atteindre et de coloniser un organe cible. La sélection de ces organes est dépendante des caractéristiques biologiques de ces derniers, notamment de la microcirculation et du microenvironnement tissulaire, et des caractéristiques des cellules tumorales qui leur confèrent un tropisme tissulaire qui leur est propre. Les dernières étapes de la progression métastatique correspondent à l'implantation des cellules tumorales extravasées dans l'organe cible sous forme de cellules isolées ou d'amas pauci-cellulaires (c'est-à-dire comprenant peu de cellules), puis à la formation de micrométastases et enfin à la formation de métastases cliniquement décelables (Figure 1) [2, 3]. De nombreuses observations et données expérimentales démontrent que le développement de métastases est un processus hautement inefficace. Ainsi, on estime que seuls $2 \%$ des cellules circulantes ou CTC (circulating tumor cells) qui survivent dans le flux sanguin sont 


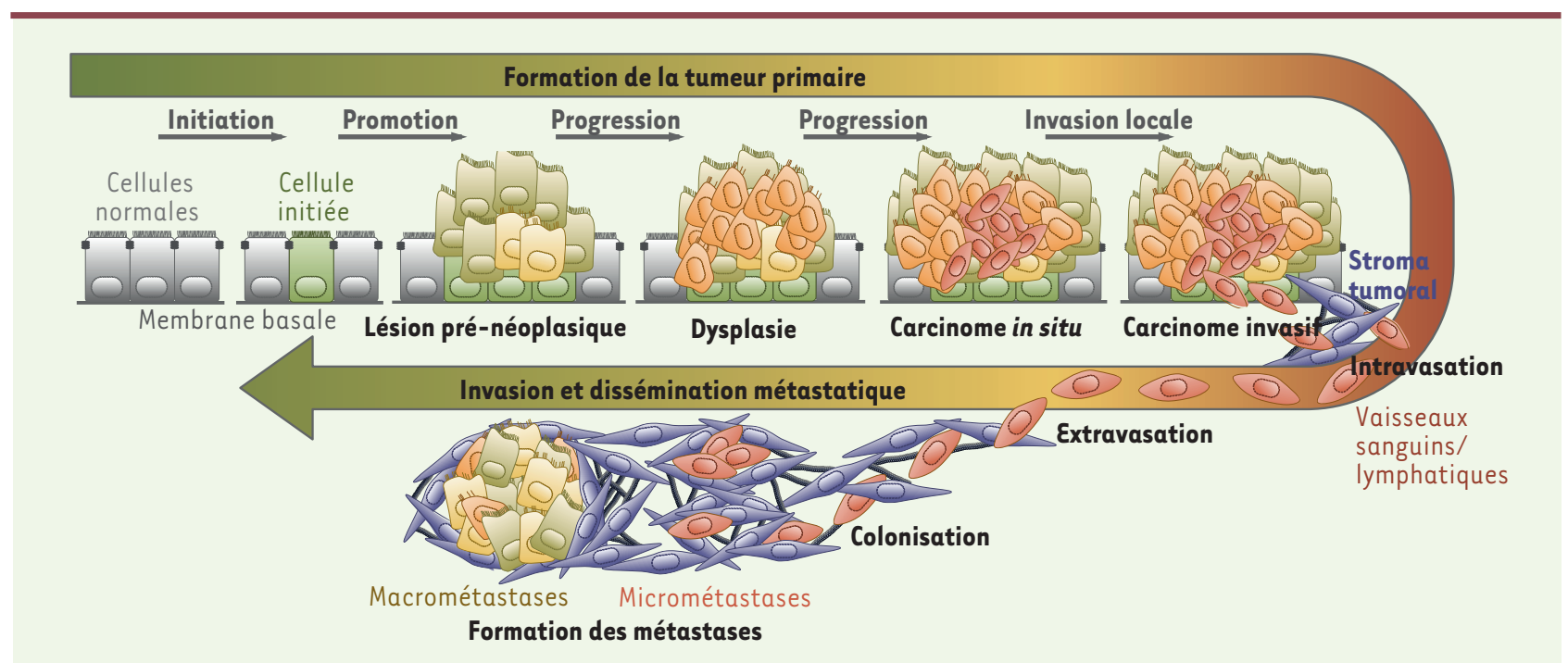

Figure 1. Principales étapes du développement d'une tumeur d'origine épithéliale et de la cascade métastatique. La formation d'une tumeur bénigne (adénome) puis maligne (carcinome) repose sur l'acquisition progressive d’anomalies génétiques et épigénétiques conférant aux cellules des capacités accrues de prolifération et de survie. La cascade métastatique est initiée par une phase d'invasion tissulaire, menant à un carcinome invasif, suivie par une phase d'intravasation, les cellules cancéreuses pénétrant alors dans la circulation sanguine ou lymphatique. La survie dans la circulation de certaines cellules malignes et leur extravasation pourront conduire, après une phase de latence, à la colonisation d'un site secondaire pour former des micrométastases puis des métastases détectables dans un site distant de celui de la tumeur primaire.

à l'origine de micrométastases et seulement $1 \%$ de ces micrométastases progresse jusqu'à un stade macroscopique [4]. En effet, la majorité des cellules qui ont réussi à entrer dans la circulation, ou à arriver dans un tissu à distance, meurent du fait de leur incapacité à s'adapter à leur nouvel environnement. Les cellules survivantes entrent pour la plupart dans un état de «dormance », caractérisé par une absence de prolifération. L'échappement à cet état de dormance est donc nécessaire au développement de métastases.

Le franchissement de chacune des étapes de la dissémination métastatique requiert des capacités uniques de motilité, d'adaptation métabolique et de survie des cellules cancéreuses. Nous proposons d'explorer le rôle de la plasticité cellulaire et du dialogue entre les cellules cancéreuses et leur microenvironnement dans ce processus complexe et d'évoquer l'importance de l'hétérogénéité intratumorale dans l'émergence de cellules à potentiel métastatique.

\section{Plasticité cellulaire et dissémination métastatique}

La dissémination métastatique a longtemps été présentée comme une phase tardive de la progression tumorale. En effet, sur la base d'une vision exclusivement génétique de la cancérogenèse, il était proposé que les étapes d'invasion et de dissémination soient initiées par l'acquisition par quelques cellules tumorales d'anomalies génétiques leur conférant des propriétés de motilité et de survie. Cependant, trois observations majeures ont conduit à réviser cette notion : le faible nombre d'événements génétiques connus pour être directement associés au processus métastatique ; l'observation de processus précoces d'invasion et de dissémination dans certains cancers; la mise en évidence de la complexité biologique du processus et du nombre de propriétés requises pour en franchir les différentes étapes. Aussi, une hypothèse mécanistique additionnelle a été proposée : la dissémination métastatique ferait appel à une reprogrammation épigénétique des cellules cancéreuses qui pourrait survenir dès les phases précoces de la progression tumorale. La réactivation de la transition épithélio-mésenchymateuse (TEM) se trouve au cœur de ce concept $[40](\rightarrow)$.

La TEM est un processus embryonnaire au cours duquel les cellules épithéliales perdent leur cohésion, $\rightarrow$ Voir la Synthèse de S. Hubert

et J.P. Abastado, $\mathrm{m} / \mathrm{s} \mathrm{n}{ }^{\circ} 4$, avril 2014, page 378 modulent leur polarité et réorganisent la structure de leur cytosquelette. Elles s'individualisent, deviennent mobiles et acquièrent des caractéristiques morphologiques et fonctionnelles de cellules mésenchymateuses. La TEM est nécessaire à plusieurs étapes critiques de la morphogenèse aboutissant à la construction du plan de l'embryon et au développement des organes. Ce processus est contrôlé par des signaux du microenvironnement et orchestré par un réseau de facteurs de transcription (FT-TEM), dont les mieux caractérisés appartiennent aux familles SNAIL, ZEB et TWIST $[5,6]$. II consiste en une reprogrammation importante de l'épigénome, impliquant notamment des changements dans la méthylation de I'ADN, des modifications post-traductionnelles des histones, des changements d'expression 
de microARN ou de IncARN (ARN longs non codants) [7, 8]. Bien que l'implication de la TEM dans la dissémination métastatique reste encore un sujet de controverse $[9,10]$, de nombreuses observations montrent, au moins dans certains types tumoraux, que sa réactivation aberrante peut constituer un événement déterminant des étapes précoces de celle-ci. En effet, des figures morphologiques de TEM associées à une surexpression des facteurs FT-TEM ont pu être mises en évidence sur le front invasif de certains carcinomes humains [11], au niveau des cellules tumorales circulantes chez les patients atteints de cancer, ainsi que dans des modèles expérimentaux de dissémination métastatique [12]. La TEM favorise ainsi l'étape d'invasion locale en conduisant à la perte des protéines d'adhésion intercellulaire, les facteurs FT-TEM se conduisant notamment comme des répresseurs de la $\varepsilon$-cadhérine ${ }^{1}$, et en initiant le réarrangement du cytosquelette. Ces événements confèrent aux cellules cancéreuses des capacités accrues de motilité et d'invasion. L'invasion est également facilitée par l'induction d'expression de métalloprotéases (MMP) permettant aux cellules tumorales de cliver les composants de la matrice extracellulaire, notamment au niveau des invadopodes. Ces structures spécialisées des cellules invasives, sont des régions de réorganisation du cytosquelette d'actine dans lesquelles se concentre une MMP (la MTl-MMP [membrane-type matrix metalloproteinase 1] ou MMP14) essentielle à l'invasion et à la dégradation de la matrice [13]. De façon importante, l'expression des FT-TEM procure aux cellules tumorales une capacité de survie accrue, en particulier en inhibant les voies onco-suppressives dépendantes de $\mathrm{p} 53$ et de $\mathrm{Rb}$ (retinoblastoma protein) $[5,14,15,41](\rightarrow)$.

Cette propriété favorise le développement de la tumeur primaire, et protège les cellules tumorales $\rightarrow$ Voir la Synthèse de $\mathbf{0}$. Albagli, $m / s n^{\circ} 10$, octobre 2015, page 869 en cours d'invasion et de dissémination contre les stress associés aux processus d'anoïkie (mort cellulaire consécutive à la perte de contacts cellules-cellules et au détachement de la matrice extracellulaire sous-jacente), d'invasion et de dissémination. Enfin, la TEM permet aux cellules cancéreuses d'acquérir des propriétés caractéristiques de cellules souches, incluant des propriétés d'autorenouvellement, favorisant leur adaptation dans un environnement différent de celui du tissu d'origine $[16,17]$.

Si les étapes initiales de la dissémination métastatique sont favorisées, et peut-être parfois initiées, par une TEM, les étapes tardives telles que l'extravasation et la colonisation du tissu hôte semblent nécessiter une transition inverse, dite mésenchymo-épithéliale (TME) $[3,18]$, apportant une explication rationnelle à l'observation selon laquelle les métastases reproduisent généralement le phénotype de la tumeur primaire. Ainsi, expérimentalement, une cellule tumorale disséminée ou DTC (disseminated tumor cell) ayant acquis un phénotype mésenchymateux doit subir une TME pour former un clone métastatique exprimant la $\varepsilon$-cadhérine [18]. Cette nécessité est probablement liée aux capacités réduites de prolifération des cellules à l'état mésenchymateux. Comme la TEM, la TME est un processus dynamique contrôlé par des facteurs protéiques (GRHL2 [grainy head-like protein

${ }^{1}$ La $\varepsilon$-cadhérine participe à l'adhérence entre les cellules épithéliales.
2 homologue], ELF3/ELF5 [E74-like factor 3/5]) et des micro-ARN (miR-200, miR-3) [3, 18, 19] (Figure 2). Ainsi, la cascade métastatique semble reposer sur une balance épithélio-mésenchymateuse, les cellules cancéreuses à même de former une métastase étant probablement celles qui présentent la plus forte plasticité phénotypique et fonctionnelle. Cette hypothèse donne une explication rationnelle à l'observation, chez les patientes atteintes de cancer du sein, de cellules circulantes présentant un phénotype mixte intégrant à la fois des caractéristiques épithéliales et mésenchymateuses [20]. Il est important de souligner, qu'audelà des propriétés intrinsèques des cellules cancéreuses, la balance épithélio-mésenchymateuse, et de façon générale la plasticité cellulaire, sont fortement dépendantes des signaux provenant du microenvironnement. Le dialogue entre les cellules cancéreuses et leur microenvironnement constitue donc une composante essentielle de la cascade métastatique.

\section{Rôle du microenvironnement dans la plasticité des cellules cancéreuses et la dissémination métastatique}

Au sein de la tumeur primaire, les cellules tumorales sont intégrées dans un environnement complexe constitué des composants de la matrice extracellulaire (MEC) et de différents types cellulaires incluant fibroblastes, cellules endothéliales, adipocytes et cellules du système immunitaire [42] $(\rightarrow)$.

La progression tumorale est le résultat d'un dialogue réciproque et dynamique entre les cellules tumorales et leur microenvironnement. Ce dernier va influencer le comportement cellulaire au tra- $(\rightarrow)$ Voir le numéro thématique Microenvironnements tumoraux : conflictuels et complémentaires, $\mathrm{m} / \mathrm{s}$ $n^{\circ} 4$, avril 2014 vers de signaux diffusibles (facteurs de croissance, cytokines) et de contraintes structurelles (molécules d'adhésion) [21]. Initialement considérée comme une structure passive de soutien, la MEC joue un rôle actif dans l'acquisition de propriétés plastiques par les cellules tumorales en modulant au travers de récepteurs d'adhérence des voies de signalisation de type IGFl (insulin growth factor 1) [25]. Une modification de la structure de la MEC telle qu'une rigidification peut induire un confinement de cellules tumorales conduisant à une reprogrammation épigénétique activant l'expression de facteurs FT-TEM. En retour, les cellules tumorales ayant subi une TEM vont modifier leur environnement. Elles libèrent en effet des facteurs solubles (facteurs de croissance, cytokines, chimiokines) qui servent de médiateurs de communication 


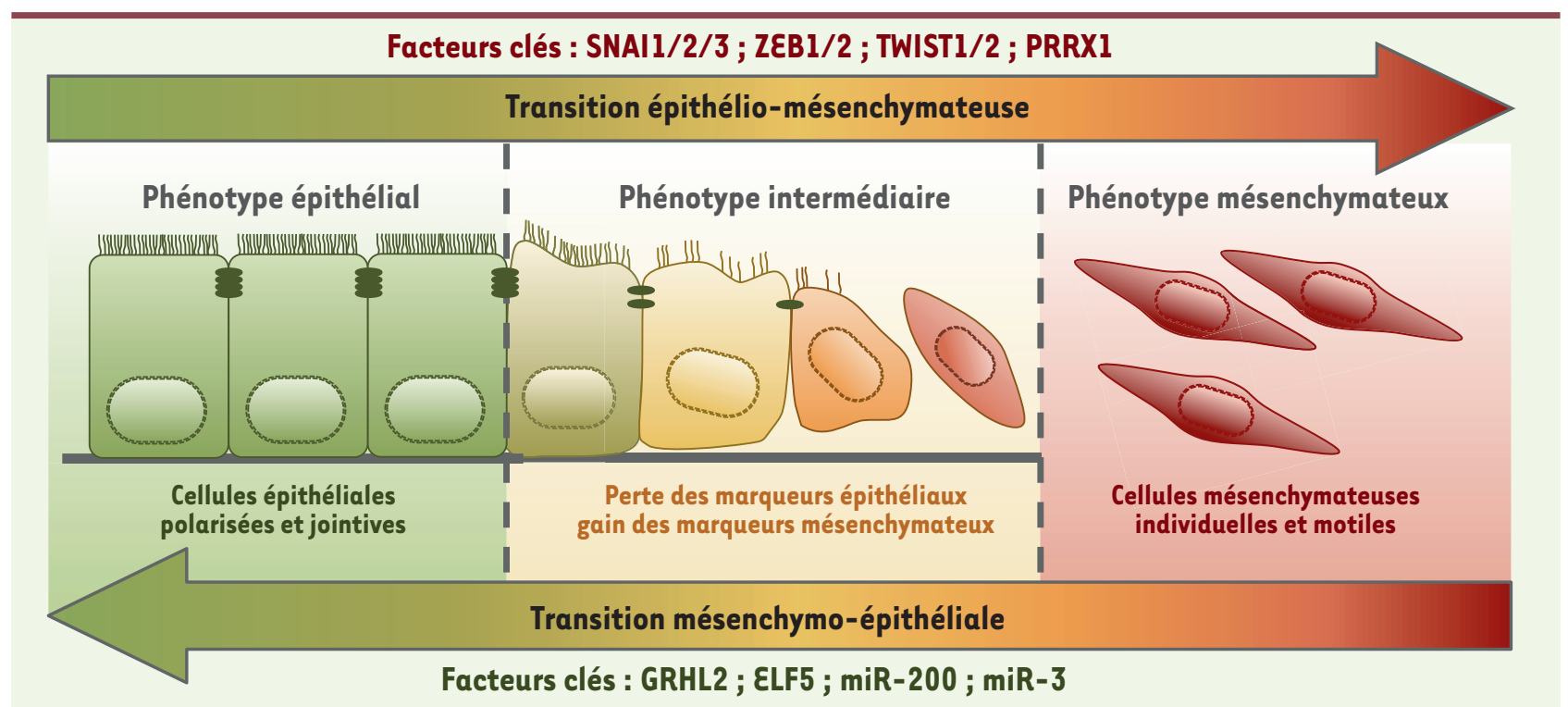

Figure 2. Transition épithélio-mésenchymateuse et dissémination métastatique. La plasticité des cellules cancéreuses joue un rôle déterminant en leur permettant de s'adapter aux contraintes qui leur sont imposées au cours des différentes étapes de la cascade métastatique. La transition épithélio-mésenchymateuse (TEM) et son processus réverse (TME) sont impliqués respectivement dans les phases précoces et tardives de cette cascade. L'expression des facteurs de transcription FT-TEM des familles TWIST, ZEB et SNAIL induit une TEM, conduisant à l'apparition de cellules mésenchymateuses motiles et invasives, alors que les facteurs GRLH2 (grainy head-like protein 2 homologue) ou ELF5 (E74-like factor 5 ) ainsi que certains micro-ARN, tels que les membres de la famille miR-200, promeuvent un phénotype épithélial.

entre cellules cancéreuses et cellules stromales environnantes, et qui favorisent la création d'un environnement apte à la génération d'une niche pro-métastatique. Les fibroblastes constituent des cellules clés de ce dialogue entre cellules cancéreuses et microenvironnement. Alors qu'ils sont relativement peu présents au sein de la matrice extracellulaire normale, ils représentent la composante cellulaire majoritaire du microenvironnement tumoral [22]. La sécrétion par les cellules tumorales de TGF $\beta$ (transforming growth factor beta) et de PDGF (platelet-derived growth factor), ainsi que le stress oxydatif associé à une activation mitogénique aberrante, favorisent, d'une part, l'initiation d'une TEM dans les cellules cancéreuses et conduisent, d'autre part, à l'activation des fibroblastes résidents [23]. Ces fibroblastes activés (appelés CAF pour cancer associated fibroblasts) vont former des myofibroblastes, qui peuvent représenter jusqu'à $80 \%$ du compartiment fibroblastique. Les CAF présentent des modifications de leur phénotype telles que l'expression de I' $\alpha$-SMA ( $\alpha$-smooth muscle actin), de la vimentine, de la desmine, de la FAP (fibroblast activated protein) ainsi que l'acquisition de capacités migratoires [24]. Ils sont capables d'interagir avec la matrice extracellulaire qui les entoure dont le remodelage qui fait appel à des protéases de types MMP ou uPA (urokinase plasminogen activator), une sérine protéase, assure, d'une part, la modification des barrières physiques qui entourent les cellules tumorales et, d'autre part, la libération de molécules séquestrées telles que I'IGF, le TGF $\beta$, Wnt et les FGF (fibroblast growth factors) [21,25] pouvant induire une TEM. L'uPA peut notamment générer une molécule active, la plasmine, ayant le potentiel de cliver des composants essentiels de la MEC que sont la laminine et la fibronectine. L'uPA induit la prolifération et la migration cellulaire et représente un facteur de mauvais pronostic dans les cancers du sein [26].

L'inflammation, fréquemment associée au processus de tumorigenèse, favorise également des interactions cellulaires qui facilitent le franchissement de certaines étapes de la dissémination métastatique. Le cancer est ainsi considéré comme une «blessure non réparée » pour laquelle l'autorenouvellement et la prolifération des cellules immunes induisent une inflammation permanente, par rétrocontrôle positif [27]. L'inflammation conduit à la production d'espèces réactives de l'oxygène, de protéases, de cytokines et de chimiokines par les cellules immunitaires. À titre d'exemple, les TAM (tumor associated macrophages) sécrètent de l'IL(interleukine)-10 et du TGF $\beta$ qui vont moduler la réponse immunitaire et activer la néo-angiogenèse [28], favorisant simultanément la plasticité cellulaire et la dissémination métastatique. La néo-angiogenèse, une angiogenèse initiée par le développement tumoral, constitue en effet un élément clé du remodelage favorisant le développement métastatique [29, 39] $(\rightarrow)$. Ce processus est initié par un déséquilibre, appelé switch angiogénique, entre facteurs $\rightarrow$ Voir la Synthèse de L. Treps et

J. Gavard, $m / s n^{\circ} 11$, novembre 2015, page 989 


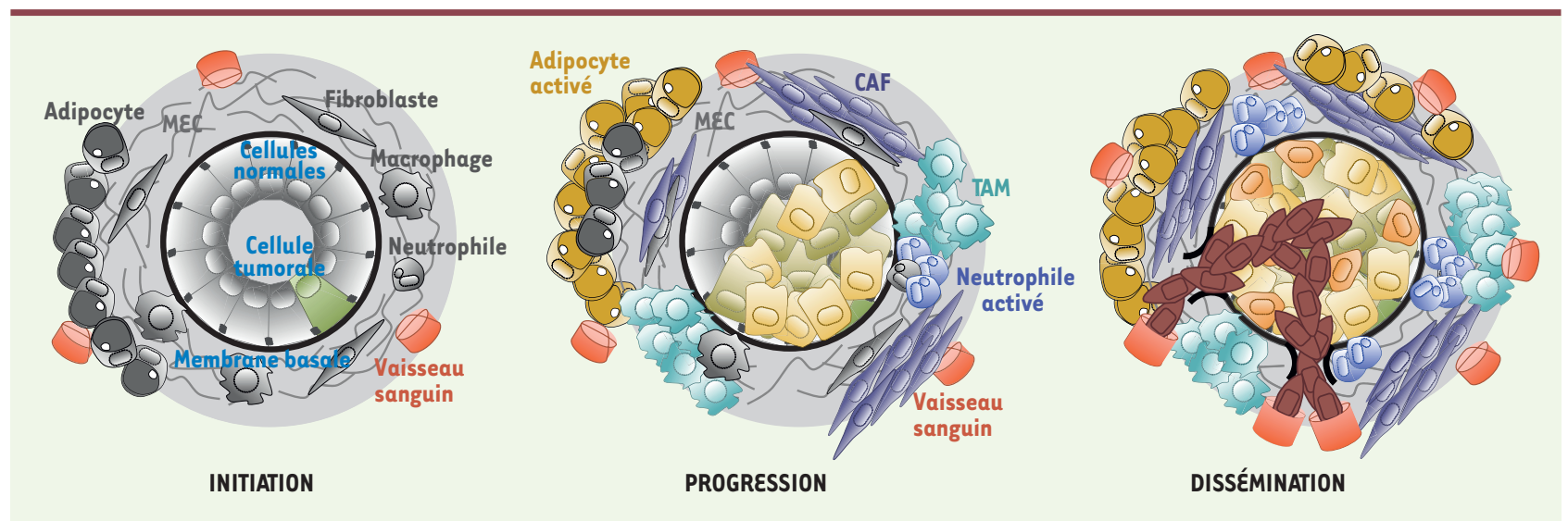

Figure 3. Rôle du stroma au cours de la progression tumorale. Le stroma et la matrice extracellulaire (MEC) subissent d'importantes modifications au cours de la tumorigenèse et de la dissémination des cellules cancéreuses. Ainsi, les monocytes recrutés au niveau de la tumeur se différencient en macrophages activés (TAM, tumor associated macrophages) et participent à la création du microenvironnement tumoral en favorisant l'angiogenèse, la migration et la croissance des cellules tumorales. L'activation des adipocytes et des fibroblastes (CAF, cancer associated fibroblasts) conduit à une augmentation de la sécrétion de facteurs de croissance, de composants de la matrice extracellulaire et de métalloprotéases facilitant le franchissement de la membrane basale, la néo-angiogenèse et le processus d'intravasation.

pro-angiogéniques (comme le VEGF [vascular endothelial growth factor], ou le FGF2 [fibroblast growth factor 2]) et anti-angiogéniques, (comme le THBS-1 [thrombospondin 1] et 2, ou I'IL-10). Les néo-vaisseaux tumoraux présentent des anomalies structurales telles qu'une membrane basale fragmentée présentant une perméabilité accrue qui favorise l'intravasation des cellules tumorales. La néo-angiogenèse fait également intervenir le facteur de transcription HIF- $1 \alpha$ (hypoxia inducible factor- $1 \alpha$ ), qui stimule l'expression de gènes cibles comme le VEGFA ainsi que certains facteurs FT-TEM, et le gène SPARC (secreted protein acidic and rich in cysteine) dont l'expression est souvent associée à une TEM. Récemment, il a été montré que la protéine SPARC était capable de favoriser l'extravasation des cellules de mélanome en provoquant l'ouverture des jonctions endothéliales vasculaires facilitant ainsi la formation de métastases pulmonaires [30].

Au total, la progression tumorale et la dissémination métastatique font appel à des interactions étroites entre les cellules tumorales et les cellules et les composants du microenvironnement (Figure 3). Certains signaux provenant du microenvironnement tumoral favorisent ainsi l'initiation d'une TEM et le franchissement d'étapes clés de la dissémination métastatique. En retour, les cellules cancéreuses peuvent modifier leur microenvironnement. Dans ce contexte, des travaux récents démontrent le rôle de la sécrétion, par les cellules tumorales, d'exosomes, des structures à double-membrane hautement spécialisées, qui sont capables de contrôler la communication intracellulaire et le microenvironnement local. Les exosomes contiennent en effet des protéines, des lipides, de l'ADN, des ARN et des microARN qu'ils peuvent transmettre après qu'ils aient fusionné avec la membrane des cellules et ainsi initier la TEM, promouvoir la néo-angiogenèse, favoriser l'échappement des cellules cancéreuses à la reconnaissance par les cellules immunitaires et participer à la formation d'une niche pré-métastatique [31, 32].

\section{Plasticité cellulaire, hétérogénéité tumorale et invasion métastatique}

La plasticité cellulaire constitue une composante essentielle de l'hétérogénéité intratumorale. Cette hétérogénéité repose sur deux phénomènes principaux. Le premier est un processus génétique basé sur la survenue de vagues successives d'expansion clonale au cours du développement tumoral. Chacune de celles-ci a pour origine l'acquisition d'une mutation conférant un avantage sélectif de croissance [33]. Ce processus est à l'origine d'une diversité génétique intratumorale accordant à la population cellulaire une forte capacité d'adaptation vis-à-vis d'un stress, au travers de la sélection de clones résistants. Le deuxième processus est de nature épigénétique, à l'origine de la plasticité phénotypique et fonctionnelle. Cette plasticité est associée à une capacité individuelle d'adaptation (Figure 4). Comme illustré précédemment par le processus de TEM, cette notion de plasticité cellulaire est intrinsèquement associée aux capacités de différenciation cellulaire. Différents modèles théoriques évoquant le rôle de la différenciation et de la plasticité cellulaires au cours de la cancérogenèse ont été établis. Le modèle hiérarchique stipule qu'au sein d'une tumeur maligne, seule une minorité des cellules cancéreuses, appelées «cellules souches cancéreuses 》 (CSC), présente des propriétés d'autorenouvellement et la capacité de régénérer une tumeur [34]. Le modèle stochastique d'interconversion repose quant à lui sur les propriétés intrinsèques de plasticité des cellules tumorales [35]. La TEM joue dans ce modèle un rôle 


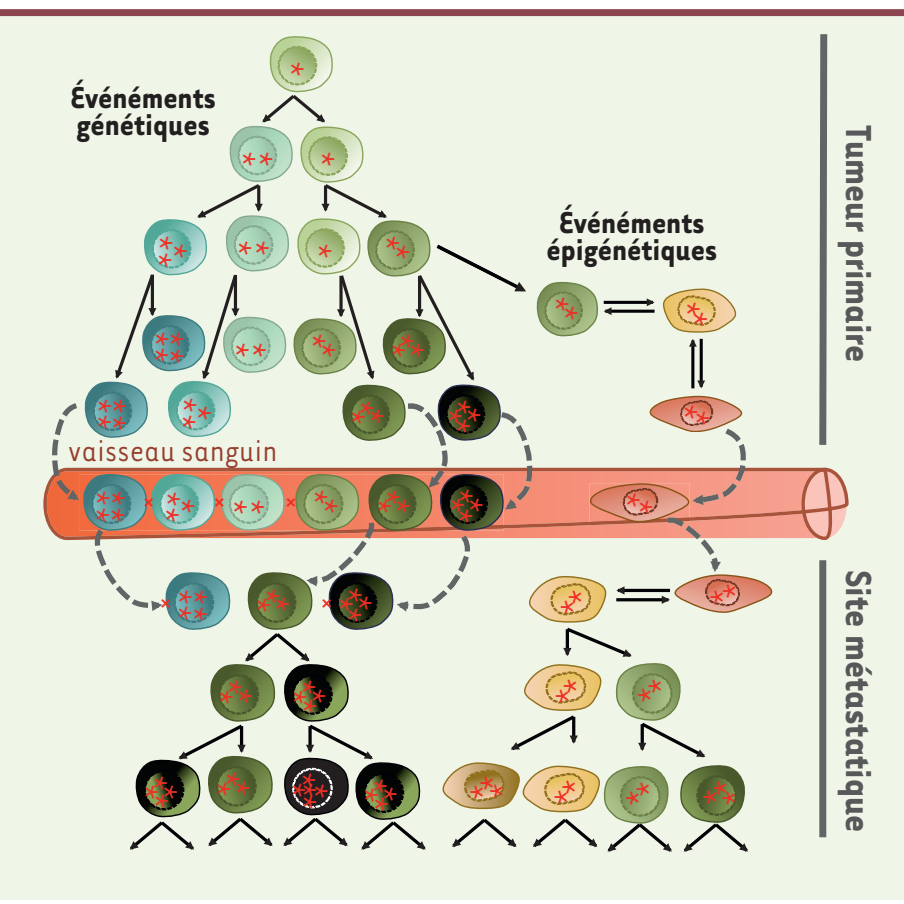

Figure 4. Hétérogénéité intratumorale et métastases. L'hétérogénéité intratumorale, d’origine génétique et épigénétique, favorise le franchissement des différentes étapes de la cascade métastatique. L'instabilité génétique associée au développement tumoral promeut une diversité génétique intratumorale et augmente la probabilité de sélection de cellules intrinsèquement résistantes aux contraintes biologiques et physiques rencontrées au cours de la dissémination (exemples de l'intravasation, de la survie dans la circulation et de l'extravasation). Dans la figure, les anomalies génétiques acquises sont représentées par une étoile $(*)$. La plasticité qui caractérise certaines cellules cancéreuses en réponse à des signaux microenvironnementaux leur confère une capacité individuelle d'adaptation, leur permettant de franchir les différentes étapes de la cascade métastatique. L'importance relative des processus génétiques et épigénétiques dans ce phénomène est probablement dépendante du type tumoral.

important, en permettant aux cellules tumorales différenciées d'acquérir des propriétés de cellules souches [16, 17].

De nombreuses observations montrent que l'hétérogénéité intratumorale et la plasticité des cellules cancéreuses soutiennent le processus métastatique en permettant l'émergence des cellules tumorales, qui présentent une résistance intrinsèque aux différentes pressions de sélection rencontrées, et des cellules aptes à s'y adapter [36]. Des modélisations mathématiques ont ainsi permis de mettre en évidence un processus darwinien de formation des métastases impliquant une évolution du phénotype cellulaire tout au long de l'invasion et de la dissémination [37]. Seules les cellules qui ont suffisamment évolué en s'affranchissant des contraintes du site primaire et qui peuvent se multiplier dans un environnement «étranger » sont aptes à générer la formation de métastases. Cette dynamique évolutive induit à la fois une divergence phénotypique entre différents clones métastatiques et une convergence phénotypique des cellules colonisant le même organe.

\section{Conclusion et perspectives}

Le développement tumoral est fréquemment considéré comme un processus d'évolution génétique dont la formation des métastases constitue la phase ultime. L'importance de l'hétérogénéité génétique intratumorale est illustrée par l'observation qu'une grande diversité clonale permet de prédire la progression d'un état pré-malin vers la malignité [38]. Cependant, au-delà de la diversité génétique, il apparaît clairement que le franchissement des différentes étapes du développement métastatique repose également sur les capacités d'adaptation des cellules cancéreuses en relation directe avec leur microenvironnement. Ainsi, le processus de TEM, mécanisme de trans-différenciation cellulaire contrôlé par des signaux du microenvironnement, peut conférer aux cellules, dès les phases précoces de la tumorigenèse, une plasticité associée à une capacité unique de motilité cellulaire, de survie et d'adaptation aux stress et aux nouveaux environnements rencontrés au cours des différentes phases de la dissémination. Remettant en question le modèle d'évolution linéaire de la progression tumorale dont le développement métastatique constitue une phase tardive et indépendante, l'initiation d'une TEM favorise, elle, la croissance de la tumeur primaire, au travers de l'acquisition de propriétés d'auto-renouvèlement et la capacité d'inhibition des voies oncosuppressives dépendantes de $\mathrm{p} 53$ et de Rb. Aussi, l'initiation de la dissémination métastatique pourrait être une conséquence secondaire d'événements nécessaires au développement de la tumeur primaire. La prévention des processus assurant une plasticité phénotypique et fonctionnelle aux cellules cancéreuses constitue donc une opportunité nouvelle pour le développement d'approches ciblant des étapes clés de la progression tumorale et capables d'empêcher l'adaptation des cellules malignes aux thérapeutiques conventionnelles. $\diamond$

\section{SUMMARY}

Cancer cell plasticity and metastatic dissemination Metastatic dissemination consists of a sequence of events resulting in the invasion by cancer cells of tissues located away from the primary tumour. This process is highly inefficient, since each event represents an obstacle that only a limited number of cells can overcome. However, two biological phenomena intrinsically linked with tumour development facilitate 
the dissemination of cancer cells throughout the body and promote the formation of metastases, namely the genetic diversity of cancer cells within a given tumour, which arises from their genetic instability and from successive clonal expansions, and cellular plasticity conveyed to the cells by micro-environmental signals. Genetic diversity increases the probability of selecting cells that are intrinsically resistant to biological and physical constraints encountered during metastatic dissemination, whereas cellular plasticity provides cells with the capacity to adapt to stressful conditions and to changes in the microenvironment. The epithelial-mesenchymal transition, an embryonic trans-differentiation process frequently reactivated during tumour development, plays an important role in that context by endowing tumor cells with a unique capacity of motility, survival and adaptability to the novel environments and stresses encountered during the invasion-metastasis cascade. $\diamond$

\section{LIENS D'INTÉRÊT}

Les auteurs déclarent n'avoir aucun lien d'intérêt concernant les données publiées dans cet article.

\section{RÉFÉRENCES}

1. Mehlen P, Puisieux A. Metastasis: a question of life or death. Nat Rev Cancer $2006 ; 6: 449-58$.

2. Thiery JP, Acloque H, Huang RY, Nieto MA. Epithelial-mesenchymal transitions in development and disease. Cell $2009 ; 139: 871-90$.

3. De Craene B, Berx G. Regulatory networks defining EMT during cancer initiation and progression. Nat Rev Cancer $2013 ; 13: 97-110$.

4. Luzzi KJ, MacDonald IC, Schmidt $\varepsilon \varepsilon$, et al. Multistep nature of metastatic inefficiency: dormancy of solitary cells after successful extravasation and limited survival of early micrometastases. Am J Pathol $1998 ; 153: 865-73$.

5. Morel AP, Hinkal GW, Thomas C, et al. EMT inducers catalyze malignant transformation of mammary epithelial cells and drive tumorigenesis towards claudin-low tumors in transgenic mice. PLoS Genet $2012 ; 8$ : el002723.

6. Puisieux A, Brabletz T, Caramel J. Oncogenic roles of EMT-inducing transcription factors. Nat Cell Biol $2014 ; 16: 488-94$.

7. Moyret-Lalle C, Ruiz $\varepsilon$, Puisieux A. Epithelial-mesenchymal transition transcription factors and miRNAs: plastic surgeons of breast cancer. World J Clin Oncol $2014 ; 5: 311-22$.

8. Richards EJ, Zhang G, Li ZP, Permuth-Wey J, et al. Long non-coding RNAs (LncRNA) regulated by transforming growth factor (TGF): LncRNA-hit-mediated TGF $\beta$-induced epithelial to mesenchymal transition in mammary epithelia. J Biol Chem $2015 ; 290: 6857-67$.

9. Fischer KR, Durrans A, Lee $S$, et al. Epithelial-to-mesenchymal transition is not required for lung metastasis but contributes to chemoresistance. Nature 2015 ; $527: 472-6$.

10. Zheng X, Carstens JL, Kim J, et al. Epithelial-to-mesenchymal transition is dispensable for metastasis but induces chemoresistance in pancreatic cancer. Nature $2015 ; 527: 525-30$.

11. Spaderna S, Schmalhofer 0 , Hlubek F, et al. A transient, EMT-linked loss of basement membranes indicates metastasis and poor survival in colorectal cancer. Gastroenterology $2006 ; 131: 830-40$.

12. Pasquier J, Abu-Kaoud N, Al Thani H, Rafii A. Epithelial to mesenchymal transition in a clinical perspective. J Oncol $2015 ; 2015: 792182$.

13. Eckert MA, Lwin TM, Chang AT, et al. Twistl-induced invadopodia formation promotes tumor metastasis. Cancer Cell $2011 ; 19: 372-86$.

14. Valsesia-Wittmann S, Magdeleine M, Dupasquier S, et al. Oncogenic cooperation between H-Twist and N-Myc overrides failsafe programs in cancer cells. Cancer Cell $2004 ; 6: 625-30$.

15. Ansieau S, Bastid J, Doreau A, et al. Induction of EMT by twist proteins as a collateral effect of tumor-promoting inactivation of premature senescence. Cancer Cell $2008 ; 14: 79-89$.

16. Morel AP, Lièvre M, Thomas C, et al. Generation of breast cancer stem cells through epithelialmesenchymal transition. PLoS One $2008 ; 3$ : e2888.

17. Mani SA, Guo W, Liao MJ, et al. The epithelial-mesenchymal transition generates cells with properties of stem cells. Cell $2008 ; 133: 704-15$.

18. Jolly MK, Boareto M, Huang B, et al. Implications of the hybrid epithelial/mesenchymal phenotype in metastasis. Front Oncol $2015 ; 5: 155$.

19. Ocaña $\mathrm{OH}$, Córcoles $\mathrm{R}$, Fabra $A$, et al. Metastatic colonization requires the repression of the epithelial-mesenchymal transition inducer Prrxl. Cancer Cell $2012 ; 22: 709-24$.

20. Yu M, Bardia A, Wittner BS, et al. Circulating breast tumor cells exhibit dynamic changes in epithelial and mesenchymal composition. Science $2013 ; 339$ : 580-4.

21. Mueller MM, Fusenig NE. Friends or foes: bipolar effects of the tumour stroma in cancer. Nat Rev Cancer $2004 ; 4: 839-49$.
22. Rønnov-Jessen L, Petersen OW, Bissell MJ. Cellular changes involved in conversion of normal to malignant breast: importance of the stromal reaction. Physiol Rev $1996 ; 76: 69-125$.

23. Scholer-Dahirel A, Costa A, Mechta-Grigoriou F. Control of cancerassociated fibroblasts function by oxidative stress: a new piece in the puzzle. Cell Cycle $2013 ; 12: 2169$.

24. Garin-Chesa P, Old LJ, Rettig WJ. Cell surface glycoprotein of reactive stromal fibroblasts as a potential antibody target in human epithelial cancers. Proc Natl Acad Sci USA $1990 ; 87: 7235-9$.

25. Lanigan F, O'Connor D, Martin F, Gallagher WM. Molecular links between mammary gland development and breast cancer. Cell Mol Life Sci 2007 ; 64 : 3159-84.

26. Duffy MJ. Urokinase plasminogen activator and its inhibitor, PAI-1, as prognostic markers in breast cancer: from pilot to level 1 evidence studies. Clin Chem $2002 ; 48$ : 1194-7.

27. Hanahan D, Weinberg RA. Hallmarks of cancer: the next generation. Cell $2011 ; 144: 646-74$.

28. Mantovani A, Sica A, Locati M. Macrophage polarization comes of age. Immunity $2005 ; 23: 344-6$.

29. Folkman J. New perspectives in clinical oncology from angiogenesis research. Eur J Cancer 1996, 32A : 2534-9.

30. Tichet M, Prod'Homme V, Fenouille N, et al. Tumour-derived SPARC drives vascular permeability and extravasation through endothelial VCAMl signalling to promote metastasis. Nat Commun $2015 ; 6: 6993$.

31. Suchorska WM, Lach MS. The role of exosomes in tumor progression and metastasis. Oncol Rep 2016 ; 35 : 1237-44.

32. Hoshino A, Costa-Silva B, Shen TL, et al. Tumour exosome integrins determine organotropic metastasis. Nature $2015 ; 527: 329-35$.

33. Nowell PC. The clonal evolution of tumor cell populations. Science 1976; $194: 23-8$.

34. Bonnet $D$, Dick JE. Human acute myeloid leukemia is organized as a hierarchy that originates from a primitive hematopoietic cell. Nat Med $1997 ; 3: 730-7$.

35. Cobaleda C, Jochum W, Busslinger M. Conversion of mature B cells into T cells by dedifferentiation to uncommitted progenitors. Nature 2007 ; 449 : 473-7.

36. Wagenblast $\varepsilon$, Soto $M$, Gutiérrez-Ángel $S$, et al. A model of breast cancer heterogeneity reveals vascular mimicry as a driver of metastasis. Nature 2015 ; 520 : 358-62.

37. Bethge A, Schumacher U, Wedemann G. Simulation of metastatic progression using a computer model including chemotherapy and radiation therapy. J Biomed Inform $2015 ; 57: 74-87$.

38. Maley CC, Galipeau PC, Finley JC, et al. Genetic clonal diversity predicts progression to esophageal adenocarcinoma. Nat Genet 2006 ; $38: 468-73$.

39. Treps L, Gavard J. L'angiogenèse tumorale. Med Sci (Paris) 2015 ; 31 : 989-95.

40. Hubert $S$, Abastado JP. Les étapes précoces du processus métastatique. Med Sci (Paris) $2014 ; 30: 378-84$.

41. Albagli 0. Protéger et sévir : p53, métabolisme et suppression tumorale. Med Sci (Paris) $2015 ; 31: 869-80$.

42. Teillaud JL. Microenvironnements tumoraux : conflictuels et complémentaires. Med Sci (Paris) $2014 ; 30: 343-462$.

43. Puisieux A, Ansieau S. La dissémination précoce de cellules métastatiques. Med Sci (Paris) $2009 ; 25: 28-9$.

\section{TIRÉS À PART}

A. Puisieux

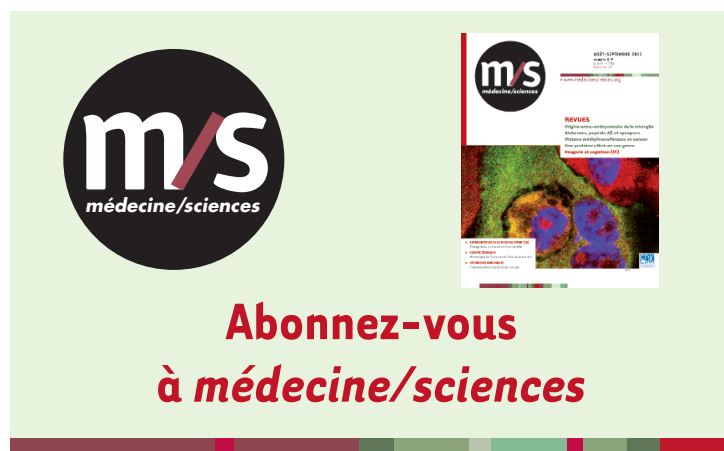

Bulletin d'abonnement page 724 dans ce numéro de $\mathrm{m} / \mathrm{s}$ 